\title{
Improving Trace Element Analysis Precision By Not Using Off-Peak Measurements
}

\author{
John J. Donovan ${ }^{1}$ and John T. Armstrong ${ }^{2}$ \\ ${ }^{1 .}$ CAMCOR, University of Oregon, Eugene, OR, 97403 \\ 2. Carnegie Institution for Science, Geophysical Lab, Washington, DC, NW, 20015-1305
}

It is well known that trace element sensitivity in Electron Probe Micro Analysis (EPMA) is limited by intrinsic random variations in the x-ray continuum background produced by the deceleration of the electron beam by the Coulombic field of the specimen atoms. Typically, this continuum must be characterized with sufficient precision (along with the peak intensity of the emission line of interest), in order to attain reasonable sensitivity for the elements of interest. Generally we characterize these intensities by measuring on either side of the emission line and interpolate the intensity underneath the peak to obtain the net intensity.

However, due to off-peak interferences from secondary emission lines, "holes" in the continuum from secondary Bragg diffraction, non-linear curvature of the wavelength dispersive spectrometer (WDS) continuum and other continuum characterization issues, this off-peak method often requires careful study of a wide swath of the emitted continuum spectrum by means of high precision WDS scans which can be quite time consuming. This is true when acquiring WDS scans with sufficient precision to detect not only the trace element emission lines of interest, but also secondary emission lines from other elements in order to avoid them when selecting off-peak measurement positions.

We will demonstrate that, at least for materials with a relatively simple matrix such as $\mathrm{SiO}_{2}, \mathrm{TiO}_{2}$ or $\mathrm{ZrSiO}_{4}$ where one may obtain suitably well characterized standards for use in the so called "blank correction" [1], we can take advantage of the Mean Atomic Number (MAN) background correction method. This technique was originally published for major and minor element characterization [2], but we will demonstrate in this paper that the MAN background method can also be utilized to obtain high precision trace element characterization without off-peak measurement by modeling the continuum absorption [3]. Trace element accuracy can be further assured by use of the previously mentioned "blank correction" technique, so that one may obtain similar accuracy with improved precision and in approximately half the acquisition time of typical off-peak trace element measurements. This MAN background method applies both to point analyses and quantitative x-ray mapping where the time savings are particularly significant and improvements in precision are especially noticeable.

Background intensities for a synthetic zircon ( $\mathrm{ZrSiO} 4)$ material calculated from traditional off-peak linear interpolation are shown fig 1, while the lower-variance backgrounds in fig 2 are from the averageZ-based MAN background calibration curve from measurements on a number of oxide standards.

[1] JJ Donovan, HA Lowers, BG Rusk (2011) Improved Electron Probe Microanalysis of Trace Elements in Quartz, American Mineralogist, 96, 274-282

[2] JJ Donovan and T Tingle (1996), An Improved Mean Atomic Number Background Correction for Quantitative Microanalysis, Jour. of Micros. Microanal., 1-7

[3] JT Armstrong (1988) Quantitative Analysis of Silicate and Oxide Materials: Comparison of Monte Carlo, ZAF, and Procedures, Microbeam Analysis, 239-246 

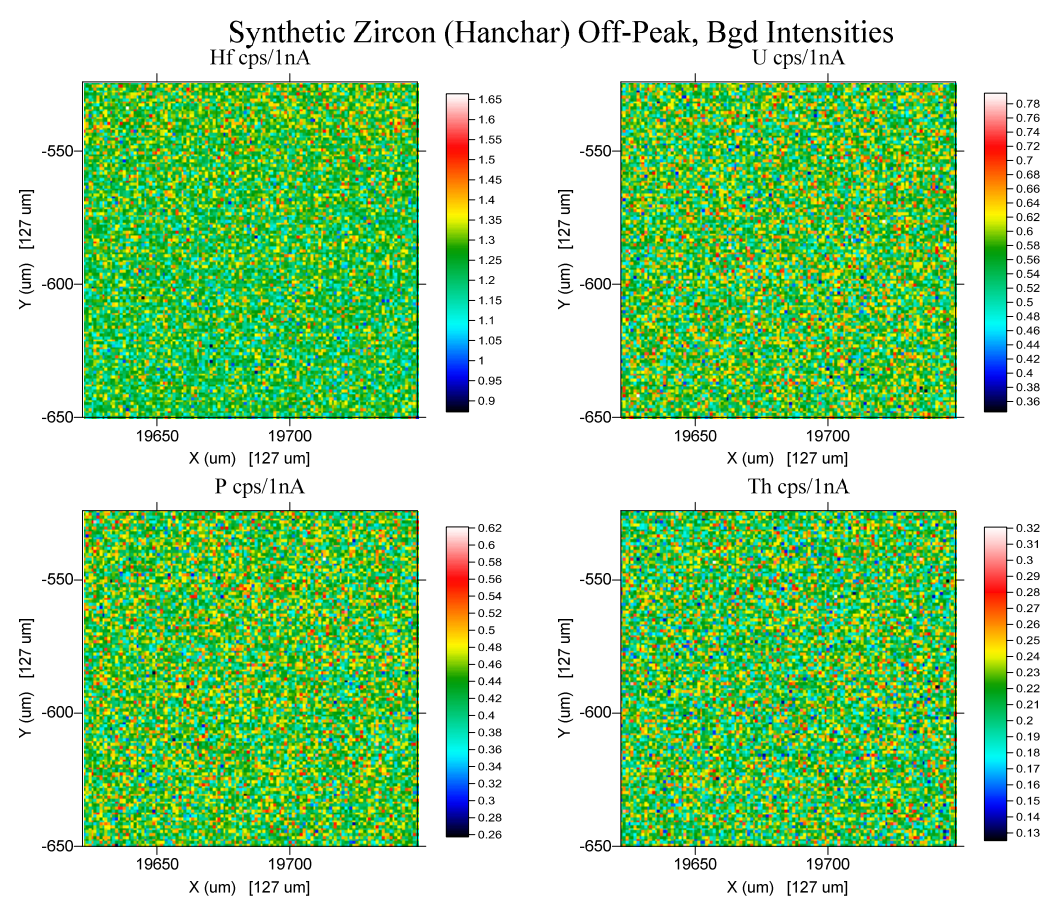

Fig.1. Calculated background intensities in a synthetic zircon using a linear interpolation from the measured off-peak intensities for Hf M $\alpha, \mathrm{U}, \mathrm{M} \alpha, \mathrm{P}$ K $\alpha$ and Th M $\alpha$. Conditions were $20 \mathrm{keV}, 100 \mathrm{nA}$, 3000 msec on-peak, 1500 msec off-peak (x2). Note that the calculated background intensities show the expected variance from the off-peak measurement uncertainties.
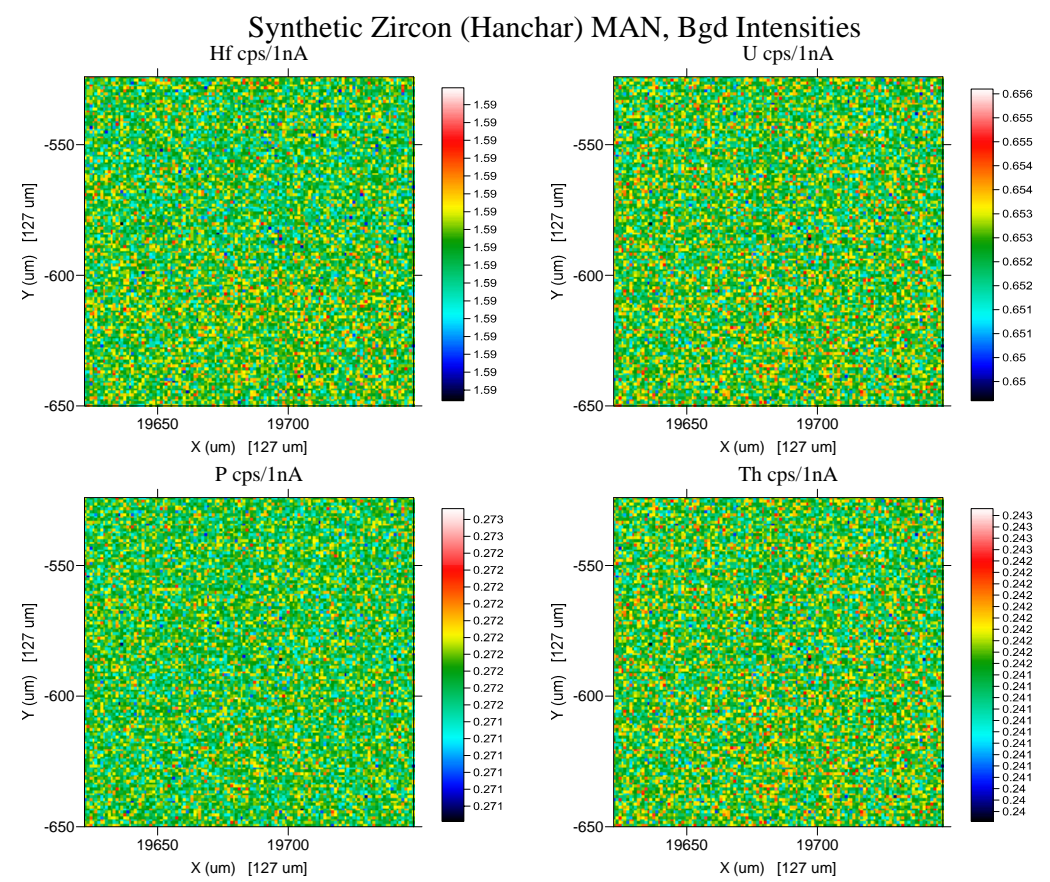

Fig.2. Calculated background intensities using a linear regression curve from the measured on-peak intensities for a number of standard materials which do not contain the elements of interest. Hf $\mathrm{M} \alpha, \mathrm{U}$ $\mathrm{M} \alpha, \mathrm{P} \mathrm{K} \alpha$ and Th M $\alpha$, at $20 \mathrm{keV}, 100 \mathrm{nA}, 3000$ msec on-peak only. Note that the calculated MAN background intensities show a much smaller degree of variance than the off-peak background intensities in Fig 1. 